This item was submitted to Loughborough's Research Repository by the author.

Items in Figshare are protected by copyright, with all rights reserved, unless otherwise indicated.

\title{
Coupling a model of human thermoregulation with computational fluid dynamics for predicting human-environment interaction
}

\section{PLEASE CITE THE PUBLISHED VERSION}

http://dx.doi.org/10.1080/19401491003615669

\section{PUBLISHER}

Taylor and Francis (@ International Building Performance Simulation Association (IBPSA))

VERSION

AM (Accepted Manuscript)

\section{LICENCE}

CC BY-NC-ND 4.0

\section{REPOSITORY RECORD}

Cropper, Paul C., Tong Yang, Malcolm J. Cook, Dusan Fiala, and Rehan Yousaf. 2019. "Coupling a Model of Human Thermoregulation with Computational Fluid Dynamics for Predicting Human-environment Interaction". figshare. https://hdl.handle.net/2134/6228. 
This item was submitted to Loughborough's Institutional Repository (https://dspace.lboro.ac.uk/) by the author and is made available under the following Creative Commons Licence conditions.

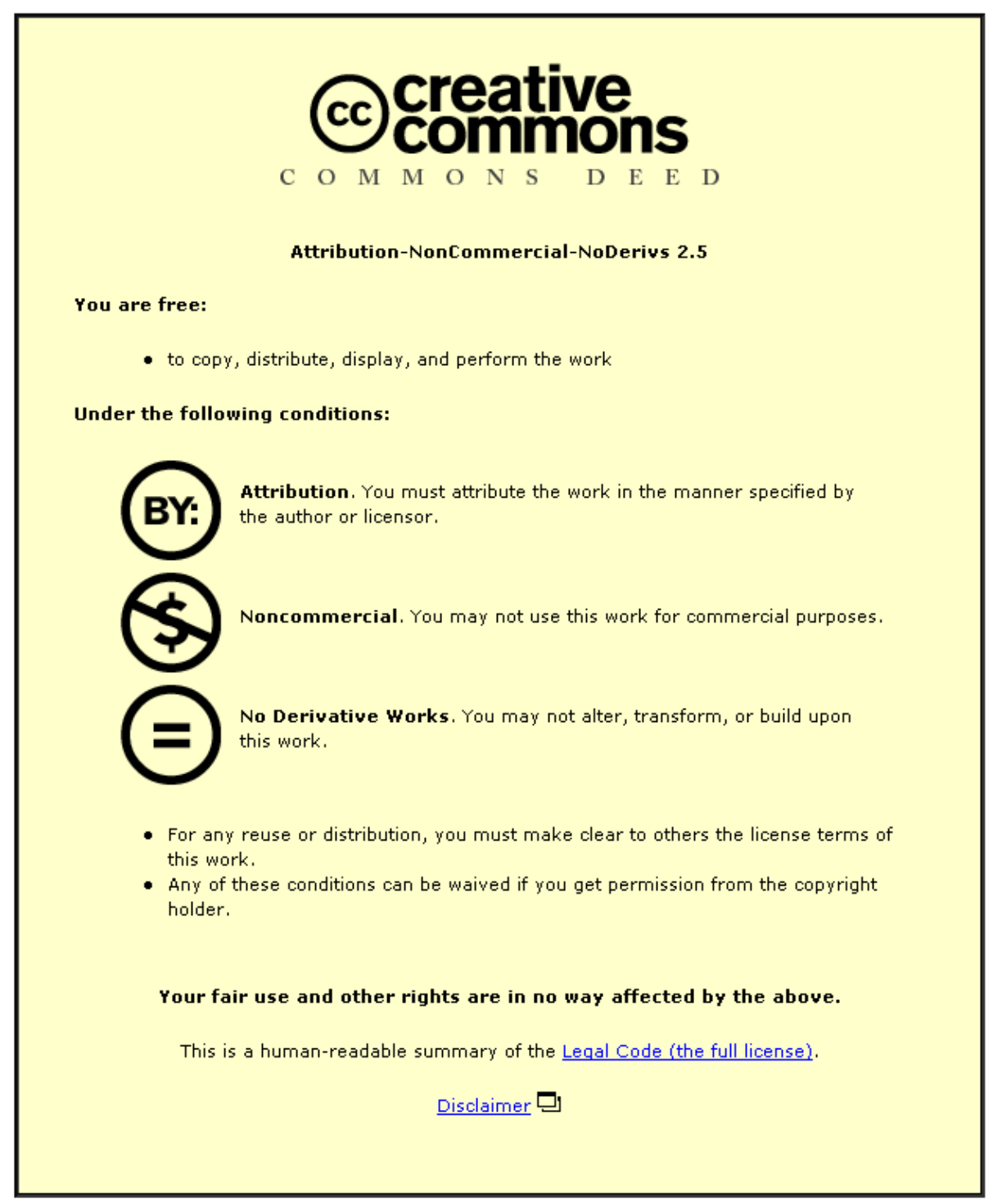

For the full text of this licence, please go to: http://creativecommons.org/licenses/by-nc-nd/2.5/ 


\section{Coupling a model of human thermoregulation with computational fluid dynamics for predicting human- environment interaction}

Paul C Cropper ${ }^{1}$, Tong Yang ${ }^{2}$, Malcolm J Cook ${ }^{2}$, Dusan Fiala ${ }^{3}$ and Rehan Yousaf $^{2}$

${ }^{1}$ Institute of Energy and Sustainable Development, De Montfort University, Leicester, UK

${ }^{2}$ Department of Civil and Building Engineering, Loughborough University,

Leicestershire, UK

${ }^{3}$ Institute for Construction Economics, University of Stuttgart, Stuttgart, Germany

Corresponding author: Dr Paul C Cropper

Dr Paul C Cropper,

Institute of Energy and Sustainable

Development

De Montfort University

Leicester

LE1 9BH

UK

Tel: +44 (0)116 2577967

Fax: +44 (0)116 2577981

Email: pcc@dmu.ac.uk

Dr Malcolm J Cook

Department of Civil and Building

Engineering

Loughborough University,

Leicestershire,

LE11 3TU

Tel: +44 (0)150922 2816

Fax: +44 (0)1509223981

Email: malcolm.cook@lboro.ac.uk

Rehan Yousaf

Hauptstrasse 60

Eichstetten Am Kaiserstuhl

79356

Germany

Tel: +49 (0)7663 4099273

Email: rehanyousaf77@googlemail.com
Dr Tong Yang

Department of Civil and Building

Engineering

Loughborough University

Leicestershire,

LE11 3TU

UK

Tel: +44 (0)1509 228799

Email: t.yang@lboro.ac.uk

Dr. Dusan Fiala

ErgonSim - Comfort Energy Efficiency

Holderbuschweg 47

70563 Stuttgart

Germany

Tel: +49 (0)7202409065

Email:dfiala@ergonsim.de

(Received 15 September 2009; final version received 30 October 2009) 
This paper describes the methods developed to couple a commercial CFD program with a multi-segmented model of human thermal comfort and physiology. A CFD model is able to predict detailed temperatures and velocities of airflow around a human body, whilst a thermal comfort model is able to predict the response of a human to the environment surrounding it. By coupling the two models and exchanging information about the heat transfer at the body surface the coupled system can potentially predict the response of a human body to detailed local environmental conditions. This paper presents a method of exchanging data, using shared files, to provide a means of dynamically exchanging simulation data with the IESD-Fiala model during the CFD solution process. Additional code is used to set boundary conditions for the CFD simulation at the body surface as determined by the IESD-Fiala model and to return information about local environmental conditions adjacent to the body surface as determined by the CFD simulation. The coupled system is used to model a human subject in a naturally ventilated environment. The resulting ventilation flow pattern agrees well with other numerical and experimental work.

Keywords: CFD; thermal comfort; model coupling

\section{Introduction}

In an attempt to provide a comfortable environment for the occupants whilst at the same time reducing energy consumption, building designers are increasingly making use of natural ventilation as an alternative to air conditioning in non-domestic buildings. By its nature, natural ventilation is less tightly controlled when compared to air conditioning, and computer modelling is often used to predict the likely performance of a building design. Computational Fluid Dynamics (CFD) is a computer modelling technique that is able to predict in considerable detail complex patterns of airflow and air temperature distribution. It has been used successfully to predict the likely ventilation performance of many advanced naturally ventilated buildings (e.g. Cook and Short 2005, Short and Cook 2005). In design practice simple shaped blocks are often used to represent human occupants in CFD models and derive empirically-based thermal comfort parameters such as PMV and PPD.

A multi-segmented human thermal comfort model (the IESD-Fiala model) has been developed that can predict the response of the human body to varying environmental conditions and can predict the resulting degree of comfort or discomfort a person experiences (Fiala et al 2003). Tanabe (2002) developed 16 body segments 65-node thermoregulation model based on probably the best known human 
thermal system model of Stolwijk (Stolwijk and Hardy 1966; Stolwijk 1971).

However, detailed heat distribution within each of the 16 segments was not modelled. The IESD-Fiala model (Fiala et al 1999) provided further refinement and improvement on the Stolwijk model and the Gagge two-node model (Gagge 1973). The IESD-Fiala model can predict the response of a human body consisting of 59 body parts (19 body segments); the model predicts dynamics thermal sensation as well as overall and local comfort or discomfort. It has been extensively validated across a wide range of steady and transient indoor and outdoor environmental conditions (Fiala et al 2001). The IESD-Fiala model uses environmental parameters, such as the temperature, humidity and velocity of air at the skin surface, to predict the response of the human thermoregulatory system to these external stimuli over a period of time.

The aim of this research is to use a commercial CFD model to predict the local environmental conditions around a human body coupled with the IESD-Fiala model to predict the response of the human body to those conditions, both the degree of comfort or discomfort experienced and temperature changes at the body surface. Changes in temperature at the body surface are also fed back to the CFD model to enable the effect that the body has on the local environment to be taken into account. This two-way data transfer is thought to be particularly important when modelling naturally ventilated spaces where air velocities are low, because the effect that a human body has on the local environment is potentially more significant than in other environments where velocities are higher.

Various degrees of coupling systems have been reported. For example Murakami et al. (2000) and Al-Mogbel (2003) used a simplified shape to represent a human body in CFD and coupled this with a two-node thermal regulatory model 
(Gagge et al 1986). Tanabe et al. (2002) integrated a 65-node human thermoregulatory model with a 3D model of a nude male body in CFD which incorporated radiation heat transfer. Using empirical heat transfer coefficients, the CFD code was used primarily to simulate the impact of the human body on the environment. Omori et al (2004) coupled a realistic nude female body with a PMV model (Fanger, 1970). Streblow et al (2008) coupled a 16-segment Tanabe model (Tanabe et al. 2002) with CFD in which local and overall thermal comfort sensations were obtained using the model of Zhang (2003). This coupled system was optimized using experimental data. Van Treeck et al (2008 and 2009) outlined a method for coupling CFD with the IESD-Fiala model (Fiala et al 2003) for predicting environmental effects on the human body, incorporating parametric body geometry with moveable limbs.

The coupled simulation system described in this paper incorporates detailed (59 body parts) and realistic human figures in CFD, combined with the IESD-Fiala model which enables the reaction of human occupants and their influence on the environment by heat and mass transfer to be modelled.

This research considers more realistic, clothed bodies in representative typical indoor environments. The research project uses CFD techniques and a long-wave radiation model to predict local environmental parameters for 59 regions of the human body and to dynamically exchange those parameters with the IESD-Fiala model to enable human thermal comfort to be predicted along with the effect the body has on its surroundings. It is expected that the coupled system will be a useful tool for researchers and some consulting engineers, enabling them to assess the impact of design decisions on occupant comfort. Consequently, a better understanding of indoor environments with improved innovative control strategies could be developed, 
providing effective design tools to improve building thermal performance, improve occupant comfort and reduce energy consumption.

This paper describes a new method for coupling CFD with the IESD-Fiala model. The system is designed using user subroutines which interact with the CFD solver. The intention is that the techniques developed will be applicable to any CFD platform which offers user-accessible development tools. The coupled system is demonstrated by modelling a person standing in a naturally ventilated environment. The resulting convective and radiative heat transfer coefficients are presented.

\section{Multi-segmented thermal comfort model}

The IESD-Fiala thermal comfort model consists of two interacting systems: the controlling active system; and the controlled passive system. The active system (Fiala et al 2001) is a cybernetic model predicting the thermoregulatory defence reactions of the central nervous system. The passive system (Fiala et al 1999) simulates the physical human body and the dynamic heat transfer phenomena that occur inside the body and at its surface.

The IESD-Fiala model also incorporates a physiologically based thermal comfort model (Fiala et al 2003) which predicts human thermal sensation responses in steady state and transient conditions.

\subsection{Active system}

A human being maintains internal temperature at a fairly constant value using four essential thermoregulatory responses; vasoconstriction, vasodilatation, shivering and sweating. Peripheral vasomotion, via suppression (vasoconstriction) and elevation (vasodilatation) of the skin blood flow, is activated to regulate internal temperature in moderate environments. In cold conditions, vasoconstriction is accompanied by shivering, i.e. a regulatory increase in the metabolic heat generation by contraction of 
muscle fibres. In warm and hot conditions, vasodilatation is accompanied by sweating, i.e. excretion of moisture at the skin which evaporates cooling the body.

The active system was developed by means of statistical regression using measured data obtained from numerous physiological experiments covering steady state and transient cold stress, cold, moderate, warm and hot stress conditions, and activity levels of up to heavy exercise.

\subsection{Passive System}

The passive system is a multi-segmental, multi-layered representation of the human body with spatial subdivisions and detailed information about anatomic and geometrical body properties. The body is idealised as 19 spherical and cylindrical elements built of annular concentric tissue layers with the appropriate thermo-physical properties and physiological functions. Tissue layers are subdivided further into spatial sectors and discretised into a total of 317 tissue nodes. The division of these elements into sectors yields a total of 59 areas at the body surface. The standard model represents an average person with a body weight of $73.5 \mathrm{~kg}$, body fat content of $14 \%$ wt, Dubois-area of $1.86 \mathrm{~m}^{2}$, basal metabolism of $87 \mathrm{~W}$, basal evaporation from the skin of $18 \mathrm{~W}$, and basal cardiac output of $4.9 \mathrm{~L} / \mathrm{min}$. The sizes and composition of the 19 body elements, including the thickness and characteristics of each of the tissue layers, are contained in a data file, enabling different body characteristics to be modelled.

Within the human body, metabolic heat is produced which is distributed over body regions by blood circulation and heat conduction from warmer to colder tissue locations.

For each sector of the passive system heat balances are established as boundary conditions at the surface. The net skin heat loss, $\boldsymbol{Q}_{s k}\left[\mathrm{~W} / \mathrm{m}^{2}\right]$ of a sector 
exposed to ambient air is equivalent to the sum of individual components of the environmental heat loss (equation 1).

$$
Q_{s k}=Q_{c}+Q_{r}+Q_{e}-Q_{s R}
$$

where $\boldsymbol{Q}_{c}\left[\mathrm{~W} / \mathrm{m}^{2}\right]$ is the heat exchange by free and forced convection with ambient air, $\boldsymbol{Q}_{\boldsymbol{r}}\left[\mathrm{W} / \mathrm{m}^{2}\right]$ the long-wave thermal radiation exchange with surrounding surfaces, $\boldsymbol{Q}_{e}\left[\mathrm{~W} / \mathrm{m}^{2}\right]$ the latent heat loss from the skin due to moisture diffusion and sweat liquid evaporation and $\boldsymbol{Q}_{s R}\left[\mathrm{~W} / \mathrm{m}^{2}\right]$ the absorption of direct and diffuse solar (short-wave) irradiation.

\subsection{Clothing model}

The IESD-Fiala thermal comfort model incorporates a relatively simple clothing model. Regions of the body covered by clothing are identified in a data file, along with the thermal characteristics of the clothing, surface emissivity and clothing area factors, enabling different levels of clothing to be modelled.

Clothing is modelled as an additional insulating layer; no attempt is made to model clothing fit or the effect of air gaps between the clothing and skin surface. Absorption of moisture by clothing is also not modelled; it is assumed that all moisture excreted by areas of the body covered by clothing appears at the clothing surface.

\subsection{Environmental heat exchange}

The IESD-Fiala model predicts the response of the human body to the local environmental conditions surrounding the body via the convective, radiative and conductive heat exchange at the body surface. In this research a CFD model is used to predict the convective heat flux $\boldsymbol{Q}_{c}$ and the long-wave radiative heat flux $\boldsymbol{Q}_{r}$ at the skin surface. In response, the IESD-Fiala model predicts the body surface temperature and evaporative heat loss flux $\boldsymbol{Q}_{e}$ resulting from the evaporation of moisture on the 
skin surface (sweating). During this ongoing research it is anticipated that the data exchanged will be extended to include the parameters required to calculate the effect of short-wave irradiation.

\subsection{Revised Body Geometry}

In this research, the simplified body geometry described above is replaced by a more realistic three-dimensional representation of a human body, termed a computational thermal manikin (CTM) (Yang et al 2007). Two fixed body geometries have been produced representing a nude figure, having 56,000 surface elements and a surface area of $1.82 \mathrm{~m}^{2}$, and a clothed figure, having 65,000 surface elements and a surface area of $2.15 \mathrm{~m}^{2}$. The manikin, illustrated in Figure 1, represents a casually dressed typical male with a height of $1.74 \mathrm{~m}$. The manikin is divided into 59 regions to match the 59 body parts of the IESD-Fiala thermal comfort model.

The manikin geometries have been optimised using surface editing and meshing tools within ICEM (ANSYS 2009), particularly in areas such as the hands, the ears, the armpits and the crotch region, to allow a CFD mesh to be constructed around them more easily (Figure 2). For example, the gaps behind the ears and between the fingers have been removed and the area smoothed to eliminate small gaps which would require an extremely fine CFD mesh and lead to increased computational load when solving the overall flow field. In the case of the clothed manikin, gaps between the clothing and body have been closed to form a 'water-tight' figure.

\subsection{IESD-Fiala model convergence}

In its stand-alone mode of operation, the IESD-Fiala model is able to predict the body's response to local environmental conditions that change over time, i.e. transient conditions. In this research, the coupled system models the interaction between the 
body and the local environment under steady-state conditions. The IESD-Fiala model is therefore run in a way that corresponds to the body being exposed to the same local conditions for a long period of time to ensure that the model reaches a steady state. In this way the IESD-Fiala model achieves internal convergence each time it is run.

\subsection{Software coding}

The IESD-Fiala model was originally written in the Delphi computer programming language, derived from Pascal, and was a 'stand-alone' program designed to run in a Microsoft DOS ${ }^{\mathrm{TM}} / \mathrm{Windows}^{\mathrm{TM}}$ environment. This research uses a version of the IESD-Fiala model translated into the platform independent Java language which allows the model to be run on a wide variety of computer platforms without the need for the code to be re-compiled into a platform specific form.

\section{CFD Model}

The commercial CFD code ANSYS CFX (ANSYS, 2007), version 11, was used to model air flow and heat transfer. Steady-state simulations have been used to model the thermal conditions in an indoor environment with a human body as the only heat source. All other surfaces were modelled as adiabatic. The CFX software employs a coupled, fully implicit solver using a transient evolution of the flow from the initial conditions. The physical timesteps used in the transient evolution provide a means of controlling the solution procedure. CFX uses a multi-element type mesh comprising hexahedrals, tetrahedrals, wedges and pyramids. The conservation equations are solved using the Finite Volume method (Versteeg and Malalasekera 1995). Flow variables (velocity, pressure, enthalpy, etc) are defined at the corners of each element which are located at the centre of each control volume used for solving the conservation equations. Solver convergence is deemed to have been achieved when 
the normalised residual values at the end of an outer iteration fall below a level specified by the user, usually $5.0 \mathrm{e}-05$ or $1.0 \mathrm{e}-05$.

The CFX software also provides a discrete-transfer long-wave radiation model which is used in the coupled simulation to predict radiative heat exchange between the body and surrounding surfaces.

\subsection{ANSYS CFX solver customisation}

ANSYS CFX was chosen for this project because it provides a powerful Application Program Interface (API) which allows additional computer code to be written to control and customise the solution process. In this research this feature is used to change the body surface temperatures and moisture during the solution cycle as predicted by the IESD-Fiala model. CFX provides a range of customisation options as follows.

\subsubsection{CFX Command Language (CCL)}

CFX Command Language (CCL) defines the controlling parameters for the simulation. In addition to standard parameters, such as the simulation boundary conditions specified by the user when setting-up the simulation, user defined control variables can also be specified. In this project, these include the upper and lower thresholds for RMS residual values and the minimum number of outer iterations between data exchanges, used by the coupling algorithm to determine if data exchange between the two models should take place. The role of these parameters is described in more detail below.

\subsubsection{CFX Expression Language (CEL)}

CFX Expression Language (CEL) is an interpreted, declarative language which can be used within the CCL to control the simulation. In this project, custom CEL functions written in FORTRAN are used to set the surface temperature and water vapour mass 
transfer for each of the 59 body parts, from tables of values supplied by the IESDFiala model.

\subsubsection{Embedded Perl}

More sophisticated customisation can be achieved through the use of embedded Perl.

Embedded Perl adds facilities to CCL such as loops, if/then/else constructs, subroutines and other common programming features. Embedded Perl can be used to implement repetitive tasks both within the solver CCL and post processing. Whilst embedded Perl can be used to implement complex control algorithms, it cannot be used within the solver execution cycle and therefore is not used in this project.

\subsubsection{User FORTRAN}

The most sophisticated customisation can be achieved using what is termed Junction Box code. Junction Box code, written in FORTRAN, provides very powerful and versatile facilities for customisation with full access to internal solver variables, subroutines and functions.

Junction Box routines, compiled to form an additional shared library, are executed in response to specific events within the solver execution cycle. The Junction Box routines to be used and the solver events they are associated with are specified within the CCL. The solver events relevant to steady-state simulations and when they occur in the solver execution cycle are illustrated in Figure 3.

In this project, Junction Box code is used to control the coupled simulation; to determine when data exchange between the two models should take place, to write heat flux values predicted by the CFX solver to an external file (to be used by the IESD-Fiala model), to read new boundary conditions from an external file (provided by the IESD-Fiala model) and to store these values to be supplied to the solver by the custom CEL functions. 


\section{Model Coupling}

The local environment that surrounds a human body (including air temperature, air speed and humidity) has an effect on the thermal state of that body and the presence of that body has an effect on the local environment that surrounds it. The IESD-Fiala model can predict the thermal state of a human body in response to a given environment and CFD can predict the local environmental conditions around a human body. By coupling the IESD-Fiala model with CFD it is therefore possible to predict human thermal comfort taking into account the two-way interaction between the body and the local environment.

In order to do this, the coupling process described in this paper and illustrated in Figure 4 uses the results predicted by each model as input conditions for the other. The IESD-Fiala model predicts 59 body surface temperatures and surface moisture, if any, due to sweating. The CFD model predicts the detailed surrounding environmental conditions including the effect the body has on that environment. As the predictions from each model have an effect on the other, the overall predictions of the coupled system are the result of an iterative process of progressive refinement.

There are two ways in which this type of coupling can be accomplished; by running models to completion each time before exchanging data (Zhang and Yang 2008) or by exchanging data while one or both of the models are running. Both of the computer models calculate a result internally through an iterative process. Whilst the IESD-Fiala model takes only a few seconds to arrive at a converged solution, the CFD model may take several hours. However, during the CFD simulation the most significant changes occur early in the solution process. The CFD model usually achieves a reasonably good state of convergence relatively quickly, spending the remaining time within a small percent of the final solution, with local conditions close to the body surface changing very little. By interrupting the CFD solution process, 
obtaining revised conditions at the body surface from the IESD-Fiala model, running the IESD-Fiala model and updating the body surface temperatures and moisture production values, it is possible to achieve convergence of the coupled system in a single, albeit longer, CFD simulation. The option of exchanging data while the CFD simulation is running was therefore adopted. However, the CFD solution process is sensitive to large changes in internal variables, including the boundary conditions at the body surface. The IESD-Fiala model is therefore run at the start of a coupled simulation, using the same initial environmental conditions as the CFD simulation, to provide an appropriate set of initial body surface temperatures and moisture production to the CFD model in order to ensure that any changes that occur at the first data exchange do not cause excessively large changes in conditions which could destabilise the solution algorithms.

\subsection{Data exchange}

Two methods of exchanging data between the models were considered; network sockets and data files. A network socket, identified by a unique (socket) number, provides a dedicated channel of communication between two computers connected via a computer network. The use of network sockets is an appropriate technique when the two computer models are to be run on different computer systems. This method has previously been successfully used to couple the IESD-Fiala model with the INKA car simulator (Fiala et al 2004). In this research the IESD-Fiala model and the CFD solver run on the same computer system. A simple approach using locally stored files is therefore employed.

\subsection{The coupling algorithm}

At the beginning of the coupled simulation, the IESD-Fiala model is run to provide an initial set of boundary conditions at the body surface, based on an initial set of 
environmental conditions. The body surface temperatures and moisture production for each of the 59 body parts predicted by the initial simulation, along with surface emissivity values, are written to a data file to be used as boundary conditions by the CFD simulation. The CFD solver is then run.

A Junction Box routine, executed in response to the User Input solver event (Figure 3) at the start of the simulation, reads initial boundary conditions from a data file created by the IESD-Fiala model. Custom CEL functions (written in FORTRAN), specified in place of fixed boundary condition values in the CCL, supply these values to the solver whenever required.

Another junction box routine, executed in response to the End of Coefficient Loop solver event (Figure 3) at the end of each outer iteration of the solver, compares the RMS residual values for pressure and momentum with the upper and lower threshold values supplied in the CCL. If all the residual values are below the upper threshold, above the lower threshold and the minimum number of iterations between data exchanges has been completed, the area averaged wall temperature, the near-wall air temperature, the convective heat transfer coefficient, the convective heat flux, the radiative heat flux and the local relative humidity for each of the 59 body parts, are written to a data file. An empty file is then produced that acts as a signal to the IESDFiala model to indicate that new environmental data is available. The CFX solver then waits for the IESD-Fiala model.

When the IESD-Fiala model detects the signal file from the CFD solver it reads the data file and performs a simulation to predict the body's response to these new local environmental conditions. When this is complete, the IESD-Fiala model writes a new set of body surface temperature and local moisture production values for each of the 59 body parts to a data file. Another empty file is then produced that acts 
as a signal to the CFX solver to indicate that new boundary condition data is available. The IESD-Fiala model then waits for the CFX solver.

When the CFX solver detects the signal file the new boundary condition data is read and solving resumed. After a data exchange has taken place, the CFX solver is run for a minimum number of iterations (specified in the CCL) before a further exchange is initiated, irrespective of the values of the residuals. This is to allow the CFD calculation process to stabilise following the change of boundary conditions.

The CFX solver and IESD-Fiala model continue to exchange data in this way until all the RMS residual values fall below the lower threshold value. No data exchange takes place while the RMS residual values remain below this threshold. However, should any of the RMS residual values rise above the threshold as the CFD solution process continues, data will again be exchanged between the two models. Data exchange is also terminated if the difference in mean body surface temperature between consecutive data exchanges is less than a pre-set threshold. The CFX solver is then allowed to run without further data exchanges taking place until the desired level of CFD convergence is achieved.

\section{Application Example}

The new coupled system has been used to model a standing male figure, dressed in casual summer clothing ( 0.6 clo), located in a naturally ventilated space with an outside air temperature of $21^{\circ} \mathrm{C}$, a relative humidity of $40 \%$ and walls with a uniform temperature of $21^{\circ} \mathrm{C}$. The computational domain has a width and depth of $3 \mathrm{~m}$ (to omit horizontal aspect ratio effects) and a height of $2.5 \mathrm{~m}$. The domain has four $0.25 \mathrm{~m} \times 0.25 \mathrm{~m}$ ventilation openings at floor level and two $0.25 \mathrm{~m} \times 0.25 \mathrm{~m}$ openings at ceiling level, all defined as free openings. This configuration is designed to have 
minimal effect on the thermal plume generated by the human in the centre of the room, the only driving force being the metabolic heat generated by the occupant.

In order to capture the flow characteristic around complicated human geometries, a turbulence model able to resolve the boundary layer near the body surface is required. The k- $\omega$ based Shear Stress Transport (SST) turbulence model (Menter 1994) was selected for its accuracy and robustness. The non-dimensional distance from the wall, $\mathrm{y}+$, must be less than 2 for sufficient accuracy to resolve boundary layer (ANSYS 2007). This was achieved using ten prism layers placed near the manikin body.

A discrete transfer radiation model was used (ANSYS 2007) to model the long-wave radiation heat exchange between the body and surrounding surfaces. The additional computational effort resulting from the use of the discrete transfer radiation model is small because the model assumes that radiation is emitted isotropically from each surface. That is, radiation is assumed to be similar in all directions. This results in a much smaller computational overhead compared to models such as Monte Carlo which is based on ray-tracing techniques.

The coupled simulation successfully predicted a buoyancy-driven natural ventilation flow driven by the heat gain generated by the IESD-Fiala model (Figure 5). The buoyant plume rising around the human body (Figure 6) feeds a layer of warm air in the upper zone of the room (Figure 7) which drives a ventilation flow out of the upper openings and in through the lower openings. The predicted flow rate of $0.31 \mathrm{ach}^{-1}$ is small as expected for such a small heat source. It can be expected that as the number of instances of the IESD-Fiala model in the CFD geometry is increased then the flow rate will also increase. The mean surface temperatures for each body part predicted by the IESD-Fiala model and used as boundary conditions in the CFD 
simulation are shown in Figure 5. As expected under these relatively benign conditions, the head is predicted to be the warmest part of the body (about $31.5^{\circ} \mathrm{C}$ ). Clothed body parts are cooler than bare flesh as they insulate the body, thereby retaining heat within the body. The predicted area-weighted convective and radiative heat transfer coefficients for all 59 body parts, calculated relative to a reference temperature of $21^{\circ} \mathrm{C}$, are given in Table 1 . The whole body mean coefficient values of $3.07\left[\mathrm{~W} / \mathrm{m}^{2} \mathrm{~K}\right]$ (convective) and $4.77\left[\mathrm{~W} / \mathrm{m}^{2} \mathrm{~K}\right]$ (radiative) agree well with published experimental data (de Dear 1997). However, direct comparison of individual heat transfer coefficients with published experimental data is not considered appropriate because of differences in the geometric subdivision of the manikins and differences in the way the heat transfer coefficients are derived.

The simulation was carried out using a computer cluster consisting of 32 nodes each equipped with dual quad-core 'Harpertown' Xeon processors and 16 GB of RAM. In this example the simulation took 7.9 CPU hours using 16 processor cores.

\section{Discussion}

Further work is needed to optimise the coupling algorithm to ensure that a solution can be obtained when modelling a wide range of environmental conditions. Simulations reported in this paper indicated that the impact of changing body surface temperatures and surface moisture production during the CFD solution process has only a small impact on the time it takes to run the CFD simulation. This approach is therefore considered to be significantly more efficient than repeatedly running each model in turn to completion. In most cases the effect of changing boundary conditions during the CFD solution process had only a temporary effect on the pattern of convergence, with the effect only lasting a small number of (CFD) iterations before the solution procedure 'recovered' and stabilised. However, simulations so far have 
been carried out modelling relatively benign environments. The effect of changing boundary conditions when simulating more challenging environments, such as those with significant asymmetries, may be greater. It is anticipated that this effect may be mitigated by ensuring that the environment used to run the IESD-Fiala model at the beginning of a coupled simulation is representative of the detailed environment modelled subsequently using CFD. However, the present system relies on the IESDFiala model's internal radiation model to calculate the radiative heat exchange during the first time the IESD-Fiala model is run because no radiation data has been obtained from the CFD radiation model at that time. This may result in large changes in the radiative heat exchange when the surface temperature and evaporative heat loss values calculated using the simple uniform internal radiation model are replaced by values calculated using the more complex radiation model in CFD if there are any significant radiative heat sources. The possibility of obtaining radiative heat exchange data from the CFD radiation model early in the coupled simulation will be investigated in the future.

Two methods of model coupling were considered in this research; running each computer model separately to completion before exchanging data or embedding one model within the solution cycle of the other and exchanging data while the containing model is running. Whilst the latter method is computationally more efficient, either method is suitable for coupling the IESD-Fiala model with a steadystate CFD simulation. However, real environmental conditions are transient, i.e. they vary with time. In a transient CFD simulation, following an initial steady-state simulation to establish the starting conditions, the results from each time step are used as the starting conditions for the next time step. Coupling the IESD-Fiala model with a transient CFD simulation would require body surface temperatures etc. to be 
updated at each time step. Using a coupling method that exchanges data at the end of the simulation would therefore be unsuitable for transient simulations. Embedding the IESD-Fiala model in the CFD solution cycle was therefore chosen because it is anticipated that future work will be based on transient simulation.

The computational thermal manikin used in this study is likely to be too complex for general use. However it was thought important in this study to use highly detailed body geometry in order to minimise any unwanted effects on air flow around the body that might result from a less smooth simple geometry. It is anticipated that the body geometry will be simplified in future work using simulation results obtained using the current complex geometry as a 'base-case' for comparison. Simplification of the body geometry and the consequent reduction in CFD mesh complexity will also enable multiple instances of the manikin to be included in the same simulation.

\section{Conclusions}

A new simulation system coupling CFD with a dynamic model of human thermoregulation and thermal comfort has been developed that is able to predict human thermal comfort in complex environmental conditions.

The use of Junction Box routines to extend the functionality of the ANSYSCFX solver has allowed specific parameters to be supplied to and extracted from the solver during the solution cycle in a form suitable for exchange with the IESD-Fiala model. The techniques described in this paper could be applied to any CFD solver, or an alternative simulation tool capable of predicting the local environment, that provides similar facilities for customisation.

It is anticipated that the use of a platform independent thermal comfort model and a simple data exchange mechanism based on text files will ensure that the techniques developed as part of this project are applicable to a wide range of 
applications, such as car, train and aircraft cabin design, as well as naturally ventilated building design.

\section{Acknowledgements}

The authors wish to acknowledge the Engineering and Physical Sciences Research

Council (EPSRC) for their support for this research through grant ref. EP/C517520/1.

The authors also wish to acknowledge the technical support provided by Dr Yehuda

Sinai and Mr Chris Staples, CFX Technical Services, ANSYS Europe Ltd.

The authors would like to thank Dr Yi Zhang, IESD, De Montfort University, for his

efforts in translating the IESD-Fiala model into the Java language.

\section{References}

Al-Mogbel A, 2003, A coupled model for predicting heat and mass transfer from a human body to its surroundings, Proc. 36th AIAA Thermophysics Conference Florida, AIAA 2003-4211.

ANSYS Inc, 2007, ANSYS CFX Help, Release 11.0, ANSYS CFX Users Guide, http://www.ansys.com

ANSYS Inc, 2009, ANSYS ICEM CFD, http://www.ansys.com/products/icemcfd.asp, accessed 8 July 2009

Cook MJ and Short CA, 2005, Natural Ventilation and Low Energy Cooling of Large, Non-Domestic Buildings - Four Case Studies, The International Journal of Ventilation, Vol. 3 No 4, pp 283-294.

de Dear, R, Arens, E, Zhang, H, Oguro, M, (1997), Convective and Radiative Heat Transfer Coefficients for Individual Human Body Segments, International Journal of Biometeorology, Vol.40:141-156

Fanger, PO, 1970, Thermal comfort. Danish Technical Press, Copenhagen, Denmark.

Fiala D, Lomas KJ and Stohrer M, 1999, A computer model of human thermoregulation for a wide range of environmental conditions: The passive system, Journal of Applied Physiology, Vol. 87 (5), pp 1957-1972.

Fiala D, Lomas KJ and Stohrer M, 2001, Computer prediction of human thermoregulatory and temperature responses to a wide range of environmental conditions, International Journal of Biometeorology, Vol. 45, pp 143-159.

Fiala D, Lomas, KJ and Stohrer M, 2003, First Principles Modelling of Thermal Sensation Responses in Steady State and Transient Boundary Conditions, ASHRAE Transactions, Vol. 109 (1), pp179-186.

Fiala D, Bunzl A, Lomas KJ, Cropper PC and Schlenz D, 2004, A new simulation system for predicting human thermal and perceptual responses in vehicles, in Schlenz D (Ed) PKW-Klimatisierung III: Klimakonzepte, Regelungsstrategien und Entwicklungsmethoden, Haus der Technik Fachbuch, Expert Verlag, Renningen, Band 27, pp 147-162, ISBN 3816922686.

Gagge AP, 1973, Rational temperature indices of man's thermal environment and their use with a two node model of his temperature regulation, Federation 
Proceedings, vol. 32, no.5, Federation of American Societies for Experimental Biology, pp 1572-1582

Gagge AP, Fobelets A, and Berglund L, 1986, A standard predictive index of human response to the thermal environment, ASHRAE Trans 92(2): 709-731.

Menter FR, 1994, Two-equation eddy-viscosity turbulence models for engineering applications, AIAA Journal 32, pp 1598-1605.

Murakami S, Kato S and Zeng J, 2000, Combined simulation of airflow, radiation and moisture transport for heat release from a human body, Building and Environment, 35(6): 489-500.

Omori T, Yang JH, Kato S and Murakami S, 2004, Coupled Simulation of Convection and Radiation on Thermal Environment around an Accurately Shaped Human Body. Proc. 9th international conference - RoomVent 2004. Portugal, pp 7.

Short CA and Cook MJ, 2005, Design Guidance for Naturally Ventilated Theatres, Building Services Engineering Research and Technology, Vol. 26, No. 3, pp 259-270.

Stolwijk JAJ, and Hardy JD, 1966,Temperature regulation in man - a theoretical study, Pflügers Archiv 291, pp 129-162

Stolwijk JAJ, 1971, A mathematical model of physiological temperature regulation in man, NASA contractor report, NASA CR-1855, Washington DC, August 1971

Streblow R, Müller D, Gores I and Bendfeldt P, 2008, A Coupled Simulation of the Thermal Environment and Thermal Comfort with an Adapted Tanabe Comfort Model, 7th International Thermal Manikin and Modelling Meeting, Portugal, http://www.adai.pt/7i3/paper.html.

Tanabe S, Kobayashi K, Nakano J, Ozeki Y and Konishi M, 2002, Evaluation of thermal comfort using combined multi-node thermoregulation $(65 \mathrm{MN})$ and radiation models and computational fluid dynamics (CFD), Energy and Buildings, 34(6): 637-646.

Van Treeck C, Pfaffinger M, Wenisch P, Frisch J, Yue Z, Egger M, and Rank E, 2008, Towards computational steering of thermal comfort assessment, Indoor Air 2008, Copenhagen, Denmark, Paper ID 762

Van Treeck C, Frisch J, Pfaffinger M, Rank E, Paulke S, Schweinfurth I, Rudolf Schwab R, Hellwig R and Holm A, 2009, Integrated thermal comfort analysis using a parametric manikin model for interactive real-time simulation, Journal of Building Performance Simulation, (in press), pp18

Versteeg HK and Malalasekera W, 1995. An introduction to computational fluid dynamics - the finite volume method, Longman Group Ltd. ISBN 0-58221884-5.

Yang T, Cropper PC, Cook MJ, Yousaf R and Fiala D, 2007, A new simulation system to predict human-environment thermal interactions in naturally ventilated buildings, Proc. 10th International Building Performance Simulation Association Conference and Exhibition (BS2007), Beijing, China, pp751-756.

Zhang H, 2003, Human thermal sensation and comfort in transient and non-uniform thermal environment. PhD thesis, University of California at Berkeley, California, USA.

Zhang Y and Yang T, 2008, Simulation of human thermal responses in a confined space, Proc. 11th Int. Conf. on Indoor Air quality and Climate (Indoor Air 2008), Copenhagen, Denmark, paper ID: 875. 
Table 1 Convective and Radiative Heat Transfer Coefficients

\begin{tabular}{|c|c|c|c|c|c|}
\hline Body part name & $\begin{array}{c}\text { hc } \\
{\left[\mathbf{W} / \mathbf{m}^{2} \mathbf{K}\right]}\end{array}$ & $\begin{array}{c}\mathbf{h r} \\
{\left[\mathbf{W} / \mathbf{m}^{2} \mathbf{K}\right]}\end{array}$ & Body part name & \begin{tabular}{|c|}
$\mathbf{h c}$ \\
{$\left[\mathbf{W} / \mathbf{m}^{2} \mathbf{K}\right]$}
\end{tabular} & $\begin{array}{c}\mathbf{h r} \\
{\left[\mathbf{W} / \mathbf{m}^{2} \mathbf{K}\right]}\end{array}$ \\
\hline Forehead & 5.48 & 5.91 & Left arm lower posterior & 3.71 & 4.87 \\
\hline Head & 3.99 & 4.84 & Right arm lower anterior & 0.35 & 4.86 \\
\hline Face anterior & 7.52 & 5.62 & Right arm lower exterior & 2.85 & 5.50 \\
\hline Left face exterior & 5.06 & 5.48 & Right arm lower inferior & 2.63 & 2.61 \\
\hline Right face exterior & 4.98 & 5.43 & Right arm lower posterior & 3.07 & 5.09 \\
\hline Neck anterior & 5.68 & 3.87 & Left hand back & 3.77 & 5.58 \\
\hline Left neck exterior & 4.32 & 4.65 & Left hand palm & 3.43 & 3.76 \\
\hline Right neck exterior & 4.14 & 4.52 & Right hand back & 3.91 & 5.66 \\
\hline Neck posterior & 3.40 & 5.09 & Right hand palm & 3.15 & 3.73 \\
\hline Left shoulder left & 2.44 & 4.97 & Left leg upper anterior & 2.79 & 5.14 \\
\hline Right shoulder right & 2.23 & 4.92 & Left leg upper exterior & 2.49 & 5.38 \\
\hline Thorax anterior & 2.98 & 5.25 & Left leg upper inferior & 2.52 & 2.27 \\
\hline Left thorax inferior & 2.69 & 3.72 & Left leg upper posterior & 2.53 & 5.13 \\
\hline Right thorax inferior & 2.74 & 3.60 & Right leg upper anterior & 3.01 & 5.07 \\
\hline Thorax posterior & 2.85 & 5.53 & Right leg upper exterior & 2.85 & 4.71 \\
\hline Abdomen anterior & 2.49 & 4.94 & Right leg upper inferior & 2.60 & 2.12 \\
\hline Abdomen left inferior & 2.34 & 4.46 & Right leg upper posterior & 2.23 & 5.32 \\
\hline Abdomen right inferior & 2.41 & 3.48 & Left leg lower anterior & 2.76 & 5.40 \\
\hline Abdomen posterior & 2.54 & 5.38 & Left leg lower exterior & 2.97 & 5.50 \\
\hline Left arm upper anterior & 2.31 & 4.84 & Left leg lower inferior & 2.97 & 3.97 \\
\hline Left arm upper exterior & 2.82 & 5.61 & Left leg lower posterior & 3.06 & 5.10 \\
\hline Left arm upper inferior & 4.50 & 0.88 & Right leg lower anterior & 3.20 & 4.99 \\
\hline Left arm upper posterior & 2.51 & 5.16 & Right leg lower exterior & 2.97 & 5.49 \\
\hline Right arm upper anterior & 1.86 & 4.99 & Right leg lower inferior & 3.08 & 3.94 \\
\hline Right arm upper exterior & 2.59 & 5.61 & Right leg lower posterior & 2.79 & 5.49 \\
\hline Right arm upper inferior & 4.61 & 1.05 & Left shoe upper & 3.62 & 4.39 \\
\hline Right arm upper posterior & 2.82 & 5.23 & Left shoe lower & 3.91 & 5.21 \\
\hline Left arm lower anterior & 1.72 & 4.63 & Right shoe upper & 3.65 & 3.95 \\
\hline Left arm lower exterior & 3.23 & 5.46 & Right shoe lower & 3.92 & 5.07 \\
\hline \multirow[t]{2}{*}{ Left arm lower inferior } & 3.60 & 2.28 & & & \\
\hline & & & $\begin{array}{l}\text { Whole body area- } \\
\text { weighted mean }\end{array}$ & 3.07 & 4.77 \\
\hline
\end{tabular}


Figure 1, Composition and subdivision of the clothed body geometry

Figure 2, The clothed body geometry within the CFD mesh

Figure 3, Events within the CFD solver solution process where junction box routines can be called

Figure 4, The model data exchange process

Figure 5, Predicted air speed around the body (left-hand legend) and predicted body surface temperatures (right-hand legend)

Figure 6, Velocity plot shows the thermal plume above the body

Figure 7, Predicted air temperatures around the body (left-hand legend) and predicted body surface temperatures (right-hand legend) 

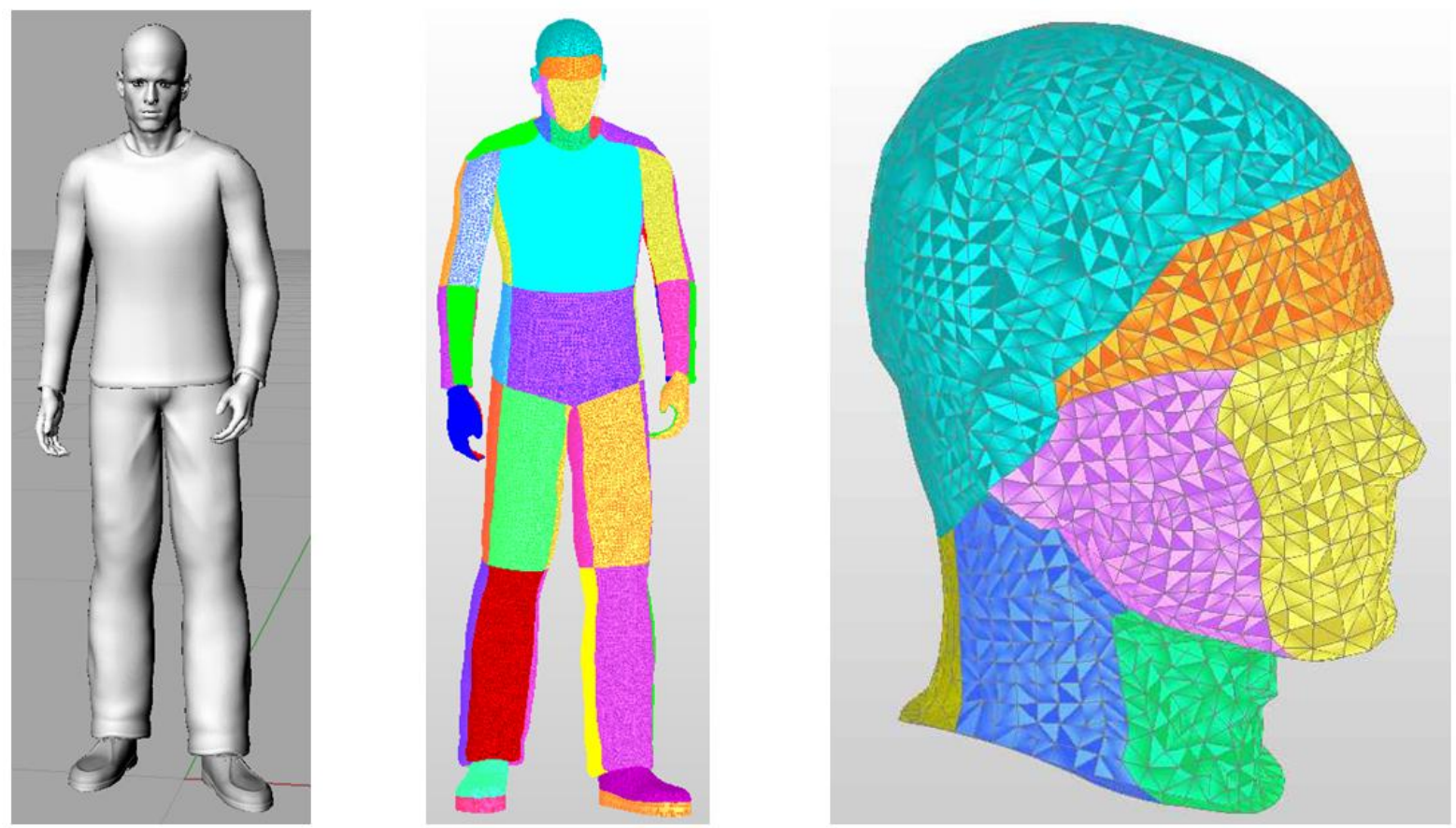

Figure 1, Composition and subdivision of the clothed body geometry 

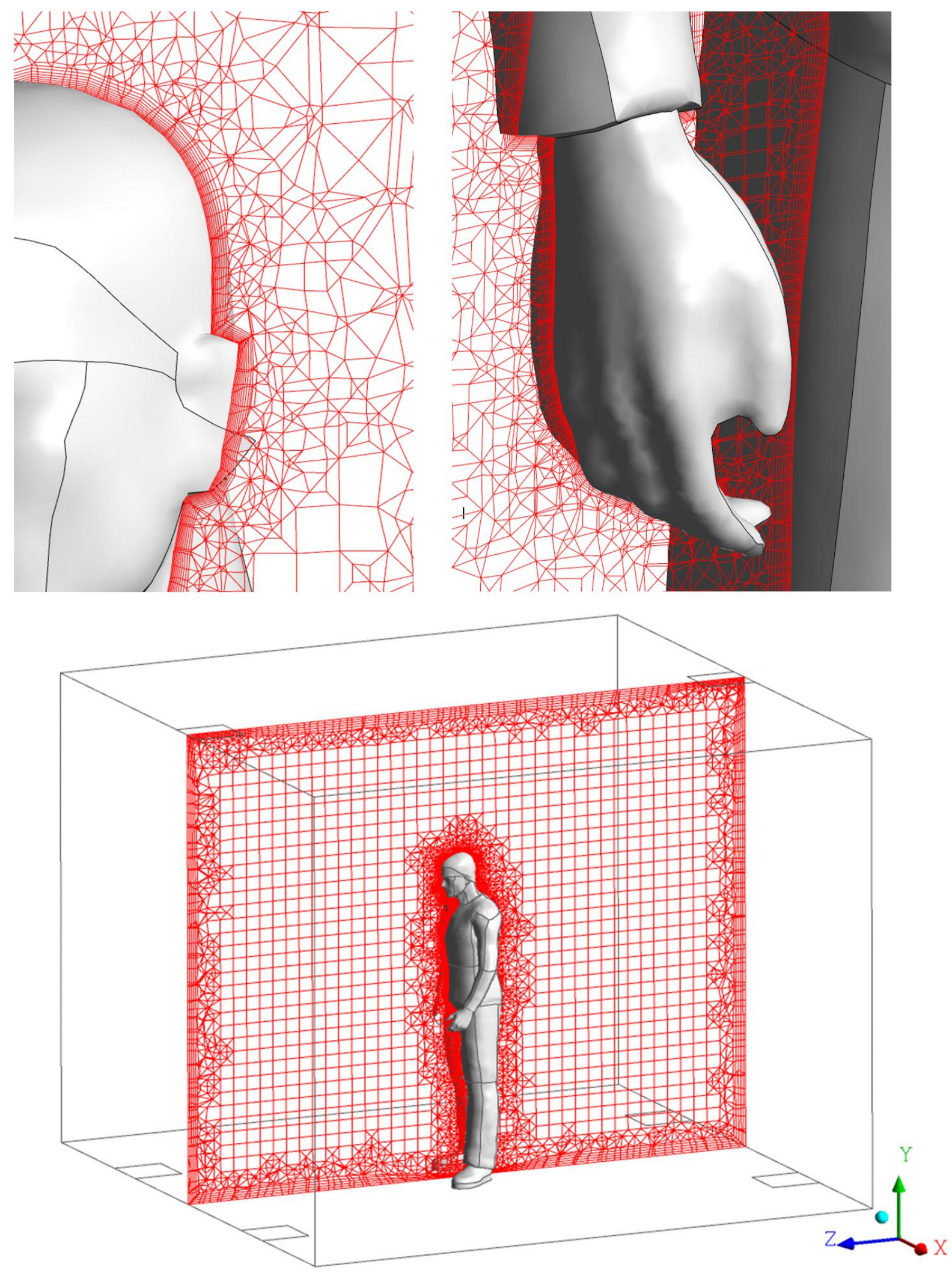

Figure 2, The clothed body geometry within the CFD mesh 


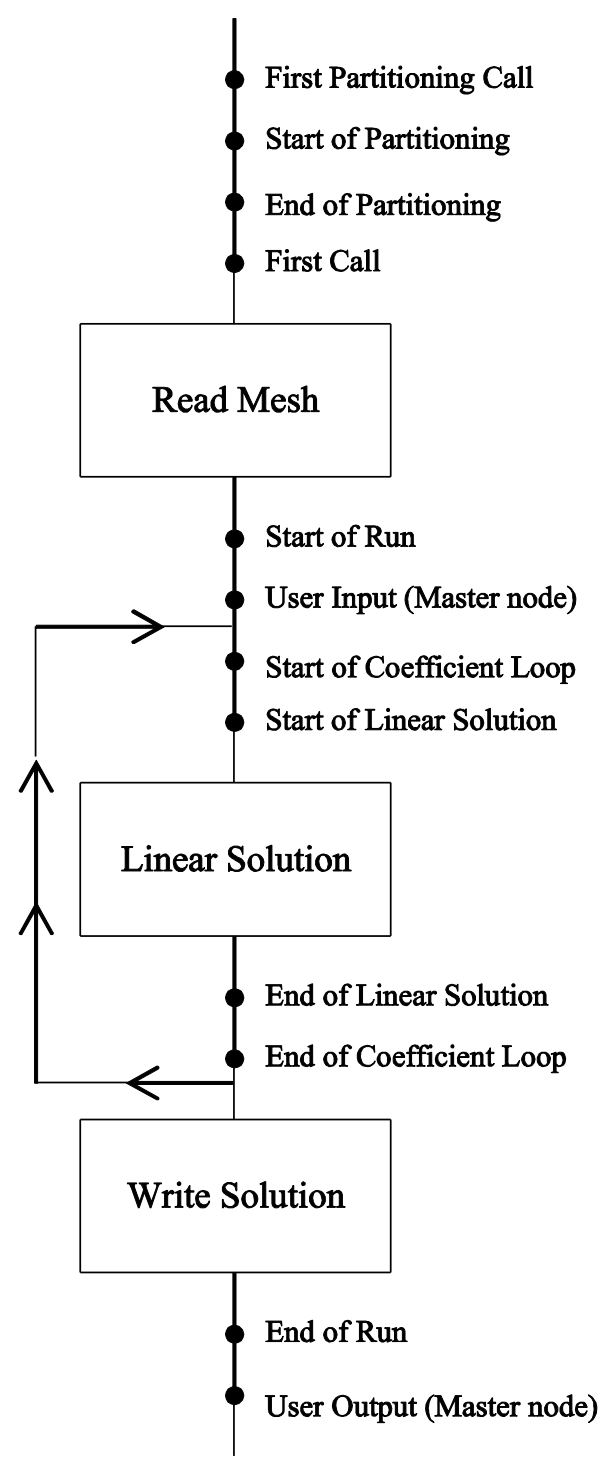

Figure 3, Events within the CFD solver solution process where junction box routines can be called 
Surface Temperature (59)

Moisture Mass fraction (59)

Surface Emissivity (59)

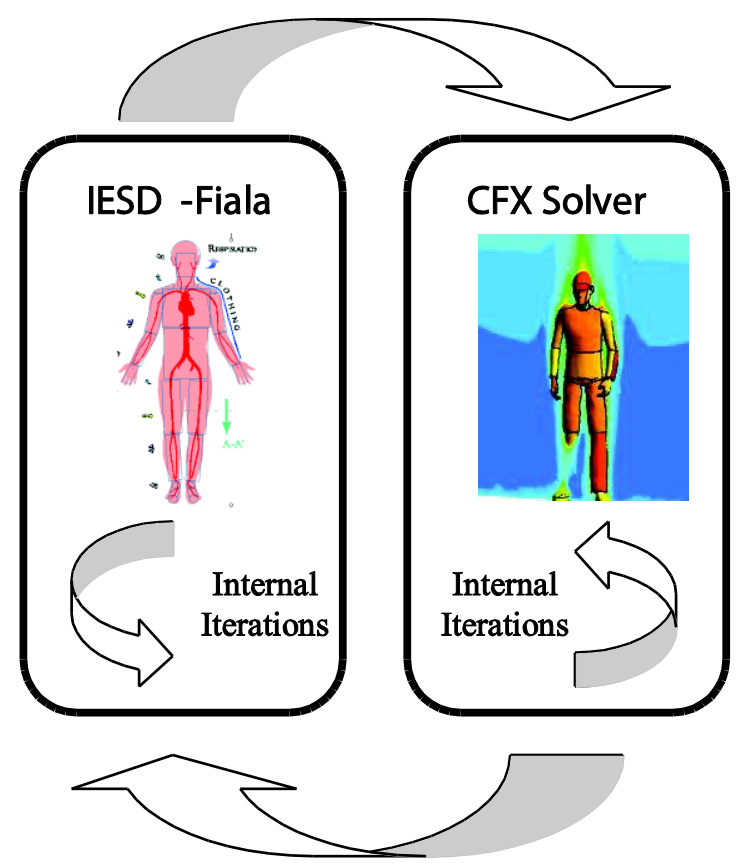

Wall temperature (59)

Near-Wall Air Temperature (59)

Convective Heat Transfer Coefficient (59)

Convective Heat Flux (59)

Radiative Heat Flux (59)

Relative Humidity (59)

Figure 4, The model data exchange process 


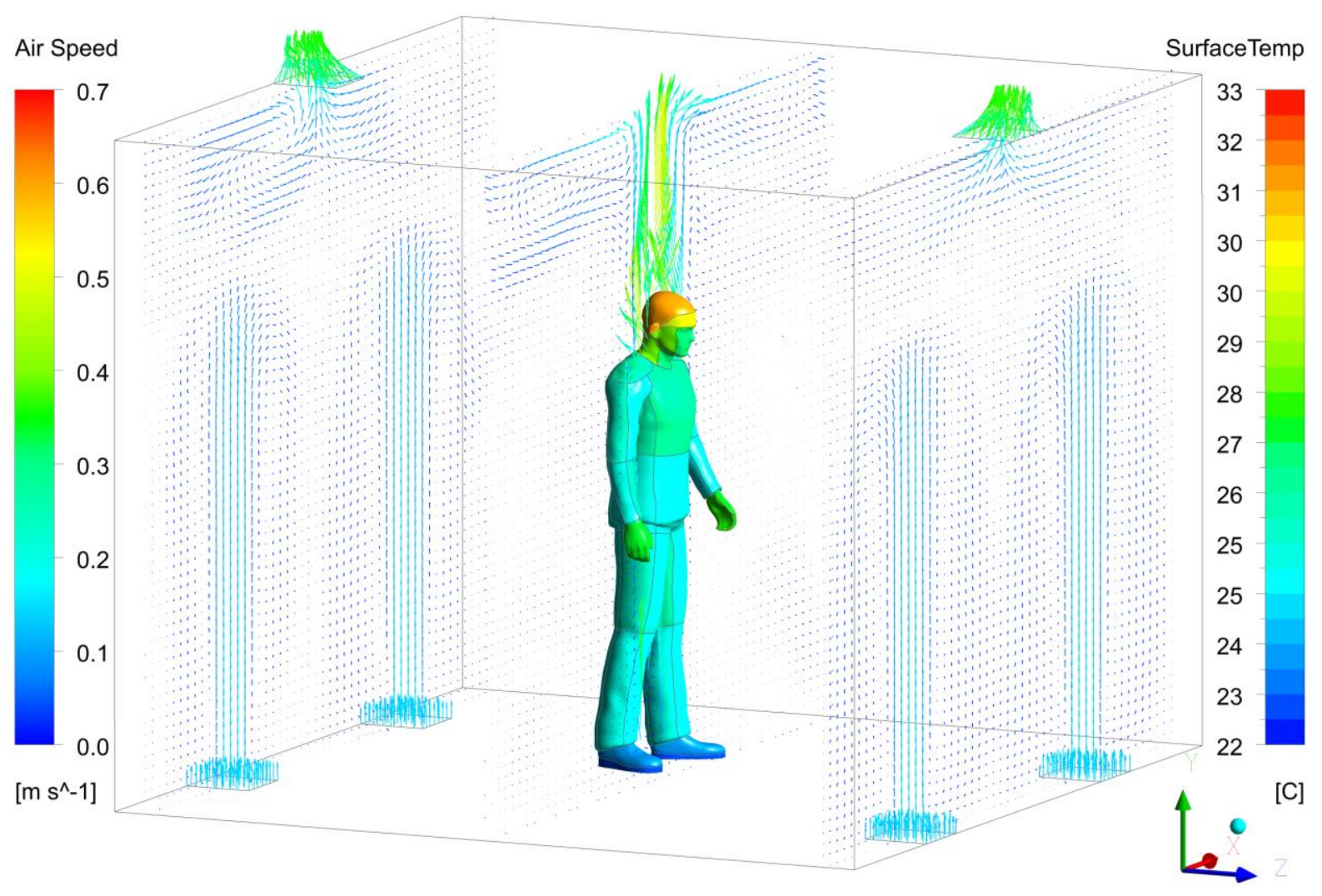

Figure 5, Predicted air speed around the body (left-hand legend) and predicted body surface temperatures (right-hand legend) 


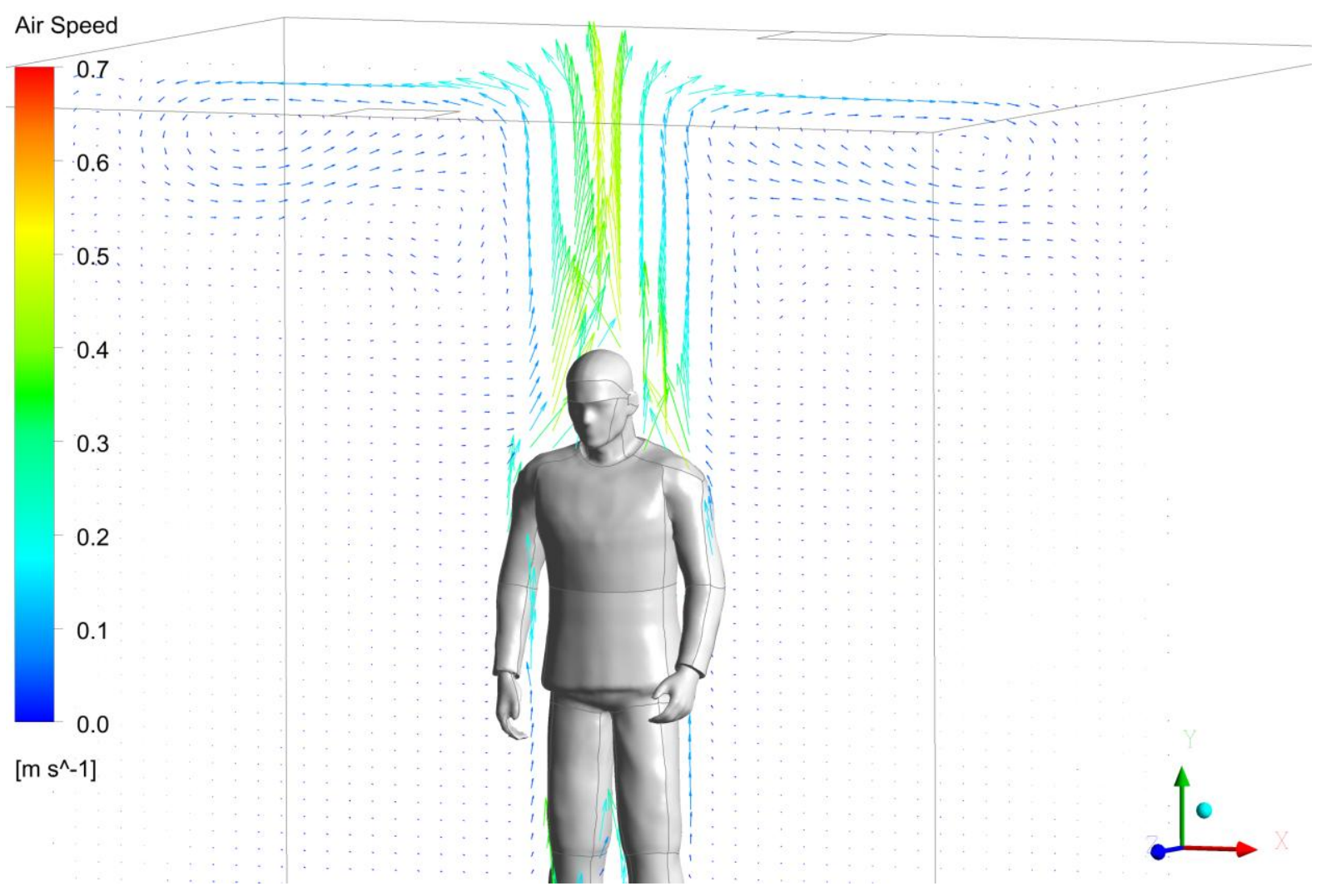

Figure 6, Velocity plot shows the thermal plume above the body 


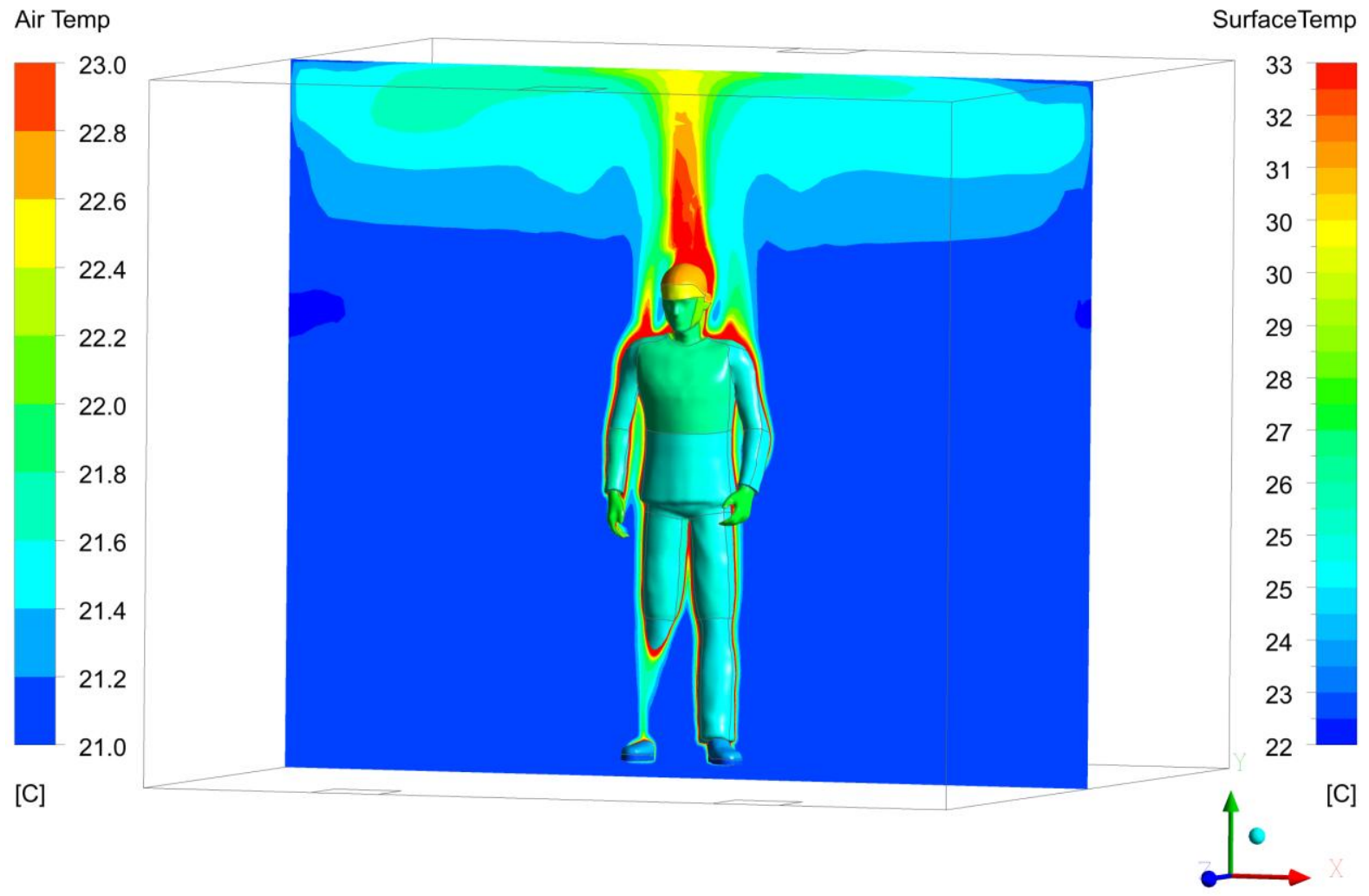

Figure 7, Predicted air temperatures around the body (left-hand legend) and predicted body surface temperatures (right-hand legend) 